\title{
La actividad física reduce el riesgo cardiovascular en mujeres diabéticas
}

Physical Activity and Cardiovascular Events in Diabetic Women. Hu FB, Stampfer MJ, Solomon C, et al. Ann Intern Med.2001; 134:96-105.

\section{Objetivo}

Determinar si la actividad física disminuye el riesgo cardiovascular en mujeres diabéticas.

\section{Diseño}

Estudio de cohortes prospectivo.

\section{Lugar}

The Nurses' Health Study fue una cohorte de 121.700 mujeres de 11 estados de EE.UU.

\section{Pacientes}

Participaron 5125 enfermeras diabéticas.

\section{Evaluación de factores pronósticos}

A través de un cuestionario validado, las participantes reportaron las horas semanales de actividad física moderada o vigorosa en 1980,1982, 1986, 1988 y 1992.

\section{Resultados}

Durante 14 años de seguimiento (31.432 pacientes/años), se hallaron 323 nuevos casos de enfermedad cardiovascular (225 casos de enfermedad coronaria y 98 casos de accidente cerebrovascular). El riesgo relativo ajustado por la edad de acuerdo al promedio de horas de ejercicio moderado ó actividad vigorosa por semana puede verse en la siguiente tabla. Los valores no cambiaron sustancialmente luego de ajustarlo por otros factores de riesgo cardiovascular.

\begin{tabular}{l|l|l}
$\begin{array}{l}\text { Ejercicio semanal promedio } \\
<1 \text { hora }\end{array}$ & RR ajuslado por edad (IC 95\%) & P de tendencia \\
\hline 1 a 1.9 horas & 1 & $<0,001$ \\
\hline 2 a 3.9 horas & $0.93(0.69$ a 1.26$)$ & \\
\hline 4 a 6.9 horas & $0.82(0.61$ a 1.10$)$ & \\
\hline 7 horas & $0,54(0.39$ a 0.76$)$ & \\
\hline
\end{tabular}

\section{Conclusión}

El incremento de actividad física para las mujeres diabéticas disminuye sustancialmente el riesgo de enfermedad cardiovascular.

\section{Comentario}

Múltiples estudios han demostrado los beneficios de la actividad física en pacientes diabéticos. Diferentes mecanismos han sido identificados ${ }^{1}$ : aumento de la captación periférica de glucosa no mediada por insulina2, aumento del número y la actividad de los transportadores de glucosa (GLUT 4) a nivel del músculo y tejido adiposo ${ }^{3}$, aumento de la síntesis de glucógeno3,4 y disminución del tejido adiposo 5 . Este estudio prospectivo de cohortes observó un efecto dosis respuesta: a mayor actividad física mayor reducción del riesgo de enfermedad cardiovascular. Las mujeres diabéticas que realizaban 4 horas/semana de ejercicio moderado ó intenso presentaron una reducción de un $40 \%$ del riesgo cardiovascular. Un estudio reciente sugiere que la falta de entrenamiento y la inactividad fueron factores predictores de mortalidad en hombres con diabetes tipo 2 en un seguimiento de 12 años ${ }^{1}$. El presente estudio con un seguimiento de 14 años y con mediciones reiteradas de la actividad física demuestra que un aumento de una actividad física moderada ó vigorosa como una caminata puede reducir sustancialmente la enfermedad coronaria y el accidente cerebrovascular en mujeres diabéticas. Dada la naturaleza observacional de este estudio, el mismo no permite establecer una relación de causalidad. Para ello debería realizarse un estudio clínico randomizado. Sin embargo, dado que han sido probados los múltiples beneficios que aporta la actividad física como contribuir al descenso de peso, mejorar el control glucémico y el perfil lipídico es probable que la asociación hallada sea causal. Por lo tanto es razonable proponer la realización diaria de una actividad física de intensidad moderada, como la caminata durante al menos 30 minutos, como uno de los pilares fundamentales en el tratamiento de pacientes con diabetes mellitus tipo 2.

Dra. Adriana Mabel Alvarez [ Servicio de Endocrinología, Metabolismo y Medicina Nuclear. Hospital Italiano de Buenos Aires ]

\footnotetext{
Referencias

1. Wei M, Gibbons LW, Kampert JB, Nichaman MZ, Blair SN. Low cardiorespiratory fitness and physical inactivity as predictors of mortality in men with type 2 diabetes. Ann Interm Med.

2000;132:605-11.

2.Goodyear LJ, Hirshman MF, Horton ES. The glucose transport system in skeletal muscle: efects of exercise and insulin. Med St. Sffects of exercise on insulin sensitivity in humans. Diabetes Care.1992;15:1690-4. Henriksson J. Influence of exercise on insulin sensitivity.J Cardiovascular Risk. 1995;2:303-9.

5. Blair SN. Evidence for success of exersice in weight loss and control. Ann Intern Med. 1993;119:702-6.
} 